\title{
Mechanism of charge transport in poly(2,5-dimethoxyaniline)
}

\author{
Rajiv K. Singh, ${ }^{\text {a) }}$ Amit Kumar, and Ramadhar Singh \\ National Physical Laboratory, Council of Scientific and Industrial Research, Dr. K. S. Krishnan Marg, \\ New Delhi 110012, India
}

(Received 26 March 2010; accepted 3 May 2010; published online 10 June 2010)

\begin{abstract}
Poly(2,5-dimethoxyaniline) (PDMA) has been synthesized by chemical oxidative polymerization technique using varying ratio of oxidants; ferric chloride $\left(\mathrm{FeCl}_{3}\right)$ and ammonium persulfate $\left[\left(\mathrm{NH}_{4}\right)_{2} \mathrm{~S}_{2} \mathrm{O}_{8}\right]$, in an inert atmosphere at low temperature $\sim 278 \mathrm{~K}$. The synthesized samples of PDMA have been characterized by Fourier transform infrared spectroscopy, scanning electron microscopy and by measuring its dc conductivity $\left(\sigma_{\mathrm{dc}}\right)$. The temperature dependence of dc conductivity of PDMA samples has been examined in the wide temperature range 6-303 K. The analysis of dc conductivity data reveals that in the temperature range $\sim 100-303 \mathrm{~K}$, the dc conductivity is predominantly governed by Mott's three-dimensional variable range hopping, however, below $100 \mathrm{~K}$ tunneling seems to dominate. Different Mott's parameters such as characteristic temperature $\left(T_{0}\right)$, density of states at the Fermi level $\left[N\left(E_{F}\right)\right]$, the average hopping distance $(R)$, and the average hopping energy $(W)$ have been estimated for all the PDMA samples and are in good agreement with the values reported earlier for other conjugated polymers. (C) 2010
\end{abstract} American Institute of Physics. [doi:10.1063/1.3443564]

\section{INTRODUCTION}

The electronic properties of conjugated polymers have acquired a growing importance in the area of modern chemistry, biotechnology, and condensed matter physics. The wide ranging applications ${ }^{1}$ of conjugated polymers depend upon the tailoring of its appropriate properties. In recent years considerable attention has been paid to the polyaniline (PANI) family of conjugated polymers because of its low cost of synthesis, easy to produce, and relatively stable properties. ${ }^{2}$ However, it is not easily soluble in common organic solvents. To improve upon the processability of PANI, its substituted forms such as poly(2-methoxyaniline), poly(methylaniline), and poly(2,5-dimethoxyaniline) (PDMA) have been synthesized. ${ }^{3-5}$ However, only PDMA has comparable conductivity to its PANI counterpart. ${ }^{5}$ Prior to any device application, the study of charge generation, and its transport in this system are very important. PANI has been extensively investigated $^{6-11}$ for its dc electrical conductivity and the experimental data has been analyzed by application of Mott's variable range hopping (VRH) model. It seems worthwhile to mention here that Mott's VRH model ${ }^{12}$ has been extensively applied to amorphous inorganic semiconductors over the last four decades with varying degree of success in polypyrrole, poly(3-decylpyrrole), polythiophene, poly(3methylthiophene), poly(3-hexylthiophene), poly(3octylthiophene), emeraldine form of PANI. ${ }^{6-11,13-20}$ The VRH mechanism is also reported ${ }^{21}$ in poly(omethoxyaniline) in the small temperature range 298-398 K. However, no report is available in literature on the temperature dependence of dc electrical conductivity of PDMA. The present paper is a report of our measurements of dc electrical conductivity in the wide temperature range 6-303 K on various samples of PDMA. We present here the analysis of our

${ }^{a)}$ Electronic mail: rajivsingh@mail.nplindia.ernet.in. data using Mott's VRH model wherein the multiphonon processes are replaced by a process in which the only contribution to the jump frequency of the polaron is due to the single phonon absorption or emission.

\section{EXPERIMENTAL}

PDMA has been synthesized by chemical oxidative polymerization technique using mixture of binary oxidants; ammonium persulfate $\left[\left(\mathrm{NH}_{4}\right)_{2} \mathrm{~S}_{2} \mathrm{O}_{8}\right]$ (APS) and/or ferric chloride $\left(\mathrm{FeCl}_{3}\right){ }^{4}$ The synthesis was carried out in acidic deionized water (DI). 2,5-dimethoxyaniline (DMA), $\mathrm{FeCl}_{3}$, and APS were purchased from Fluka, Sigma-Aldrich and Ranbaxy Laboratories, respectively, and used as such without any further purification. Hydrochloric acid was used to acidify the Milli-Q DI. The monomer DMA was added in this acidic aqueous solution at low temperature $\sim 278 \mathrm{~K}$ in an inert atmosphere $\left(\mathrm{N}_{2}\right.$ atmosphere) and stirred for $30 \mathrm{~min}$. This mixture of binary oxidant APS : $\mathrm{FeCl}_{3}$ was added in the reaction mixture containing DMA monomer. The reaction mixture was again stirred for $3 \mathrm{~h}$ at low temperature $\sim 278 \mathrm{~K}$ in an inert atmosphere for complete polymerization of the monomer. Then this mixture was kept in vacuum oven at $\sim 358 \mathrm{~K}$ for overnight drying. The residual after overnight drying contains excess of oxidant and unreacted monomer along with desired PDMA, so the purification was done in order to get pristine polymer. The unreacted monomers, oligomers, and oxidants were removed from the polymer by successive washings by those chemicals, which show specific affinity for the molecules to be removed. The excess intercalated oxidant trapped in PDMA was further removed by solvent extraction process. Then the polymer was dried at $\sim 358 \mathrm{~K}$ for $15 \mathrm{~min}$ and the resultant polymer was obtained in black powder form. The above mentioned synthesis of PDMA was carried out by varying the ratio of binary oxi- 
TABLE I. Percentage of APS and $\mathrm{FeCl}_{3}$ in binary oxidants, their dc conductivity $\left(\sigma_{\mathrm{dc}}\right)$, and activation energy $\left(E_{A}\right)$ values at room temperature $(\sim 300 \mathrm{~K})$ for the various samples of PDMA.

\begin{tabular}{|c|c|c|c|c|}
\hline $\begin{array}{l}\text { Sample } \\
\text { number }\end{array}$ & $\begin{array}{c}\text { APS } \\
\text { (wt } \%)\end{array}$ & $\begin{array}{l}\mathrm{FeCl}_{3} \\
\text { (wt \%) }\end{array}$ & $\begin{array}{c}\text { de conductivity }\left(\sigma_{\mathrm{dc}}\right) \\
\text { at } 300 \mathrm{~K} \\
(\mathrm{~S} / \mathrm{cm})\end{array}$ & $\begin{array}{c}\text { Activation energy } \\
\left(E_{A}\right) \\
\text { at } 300 \mathrm{~K} \\
(\mathrm{eV})\end{array}$ \\
\hline PDMA1 & 0 & 100 & $4.36 \times 10^{-8}$ & 0.551 \\
\hline PDMA2 & 25 & 75 & $2.04 \times 10^{-7}$ & 0.466 \\
\hline PDMA3 & 50 & 50 & $9.28 \times 10^{-7}$ & 0.333 \\
\hline PDMA4 & 75 & 25 & $5.55 \times 10^{-8}$ & 0.351 \\
\hline PDMA5 & 100 & 0 & $7.99 \times 10^{-9}$ & 0.610 \\
\hline
\end{tabular}

dants; $\mathrm{FeCl}_{3}$ and APS, the ratio is given in Table I. Five sets of samples were prepared and are designated as PDMA1, PDMA2, PDMA3, PDMA4, and PDMA5.

The rectangular pellets $\left(5 \times 10 \mathrm{~mm}^{2}\right)$ of PDMA having thickness $\sim 1-2 \mathrm{~mm}$ were made after grinding and pressing the polymer powder at a pressure of $\sim 5 \times 10^{8} \mathrm{~Pa}$ in a hydraulic press. For the dc conductivity measurement, gold electrodes were vacuum $\left(\sim 10^{-6}\right.$ Torr $)$ evaporated on both sides of these five samples of PDMA, thus making an $\mathrm{Au} /$ polymer/Au sandwich structure. ${ }^{16,22}$ Fourier transform infrared (FT-IR) spectra of PDMA1, PDMA2, PDMA3, PDMA4, and PDMA5 were recorded on Perkin Elmer FT-IR spectrometer in the range $4000-400 \mathrm{~cm}^{-1}$. Scanning electron microscopy (SEM) of these samples was performed by using Leo 440 and Zeiss EVO MA-10 scanning electron microscope. The room temperature $(\sim 300 \mathrm{~K})$ dc conductivity $\left(\sigma_{\mathrm{dc}}\right)$ values (measured by 6517A Keithley Electrometer) of all the samples are reported in Table I. The temperature dependence of dc conductivity of these samples was measured in a closed helium cryostat (ARS USA) with LakeShore 331 temperature controller in the range $6-303 \mathrm{~K}$ by using Keithley 6517 A Electrometer.

\section{RESULTS AND DISCUSSION}

FT-IR spectra of all the samples of PDMA show the key absorption bands in the region $1500-1600 \mathrm{~cm}^{-1}$ and $1400-1500 \mathrm{~cm}^{-1}$ and are assigned to the quinoid and benzenoid ring units, respectively. The detailed discussion on FT-IR observations has been reported in our recent work. ${ }^{23}$

Figures 1(a)-1(e) shows the surface morphology of all the five samples of PDMA. It is evident from Fig. 1(a) that the sample (PDMA1) synthesized with $\mathrm{FeCl}_{3}$ oxidant is devoid of any structural ordering showing nearly amorphous character. However, samples (PDMA2, PDMA3, PDMA4) prepared with varying amounts of APS in binary oxidant $\mathrm{FeCl}_{3}$ :APS [Figs. 1(b)-1(d)] shows the growth of micron size grains in their amorphous matrix. The larger grains appear to be composed of thin (nearly two-dimensional) sheets of polymer stacked together. The ratio of APS and $\mathrm{FeCl}_{3}$ seems to play an important role in determining the morphology and crystallinity of PDMA. The largest grains are observed in the PDMA3 polymer synthesized with (50:50) ratio of binary oxidant $\mathrm{FeCl}_{3}$ :APS. Figure 1(e) shows that the amorphous regions are minimum in PDMA5, depicting growth of some needle type grains of varying dimensions in addition to micron size grains. The surface morphological results agree well with the $\mathrm{x}$-ray diffraction results ${ }^{23}$ obtained on these samples.

It is seen in Table I that the highest conductivity $\sim 9.44 \times 10^{-7} \mathrm{~S} / \mathrm{cm}$ is observed for PDMA3 sample synthesized by using $\mathrm{FeCl}_{3}$ :APS (50:50) and it decreases on moving to either side of the binary oxidant ratio. The dc conductivity value for PDMA synthesized by only $\mathrm{FeCl}_{3}$, i.e., PDMA1 is $\sim 5.84 \times 10^{-8} \mathrm{~S} / \mathrm{cm}$ and is higher than that synthesized by only APS, i.e., PDMA5 $\sim 7.72 \times 10^{-9} \mathrm{~S} / \mathrm{cm}$. The amorphous region evidenced by SEM investigations [Figs. 1(a)-1(e)] could be related to formation of spinless bipolarons in PDMA favoring the formation of quinoid structure. ${ }^{24}$ At the same time it restricts the mobility of charge carriers, whereas, the partial/little crystallinity, shows a little ordering in the polymer chain which could be attributed to the benzenoid structure where the polaronic lattice supports the mobility of charge carriers and hence, a good conductivity of the polymer PDMA. It has been reported ${ }^{10,24}$ in literature that the magnitude of dc conductivity increases with increase in number of benzenoid ring units in PDMA back-bone. The dependence of room temperature dc conductivity on the ratio of binary oxidants indicates (Table I) that the extent of benzenoid structure is maximum in sample PDMA3, which has higher conductivity than other samples of PDMA. ${ }^{23}$

In general, the dc conductivity $\left(\sigma_{\mathrm{dc}}\right)$ of conjugated polymers can be assumed to consist of three components, $\sigma_{B}, \sigma_{H}$, and $\sigma_{T}$, i.e., $\sigma_{\mathrm{dc}}=\sigma_{B}+\sigma_{H}+\sigma_{T}$, where $\sigma_{B}$ is the contribution to total conductivity due to intrachain transport of charge carriers usually described by the band conduction mechanism and is normally observed at high temperatures. $\sigma_{H}$ is the contribution to the total conductivity $\left(\sigma_{\mathrm{dc}}\right)$ due to interchain transport of charge carriers, i.e., hopping of charge carriers between the polymer chains and is observed at intermediate temperatures. $\sigma_{T}$ is the conductivity observed at low temperature and is due to thermally activated tunneling process. ${ }^{12,16,20}$ Hence in the present investigation the total observed dc conductivity $\left(\sigma_{\mathrm{dc}}\right)$ of PDMA may be correlated with the conductivity $\sigma_{H}$ for the hopping of charge carriers between the chains from room temperature $(\sim 303 \mathrm{~K})$ down to $100 \mathrm{~K}$ and below it the conductivity $\left(\sigma_{T}\right)$ is associated with the tunneling process. ${ }^{16,17}$

A decrease in the conductivity with a decrease in temperature has been observed in all the samples of PDMA. The variation in dc conductivity $\left(\sigma_{\mathrm{dc}}\right)$ as a function of $1000 / T$, in the wide temperature range of 6-303 K for PDMA1, PDMA2, PDMA3, PDMA4, and PDMA5 is shown in Fig. 2. It is evident from this figure that the dc conductivity tends to saturate in the low temperature region i.e. below $\sim 100 \mathrm{~K}$.

The activation energy evaluated from Fig. 2 has been plotted as a function of temperature in Fig. 3(a). The temperature dependence of activation energy $\left(E_{A}\right)$ [Fig. 3(a)] rules out the applicability of the band conduction for a temperature independent band gap. ${ }^{12,13}$ Another possibility could be the temperature dependent energy gap $E_{g}$. In such a situation the activation energy $E_{A}$ can be defined ${ }^{12,13}$ as 

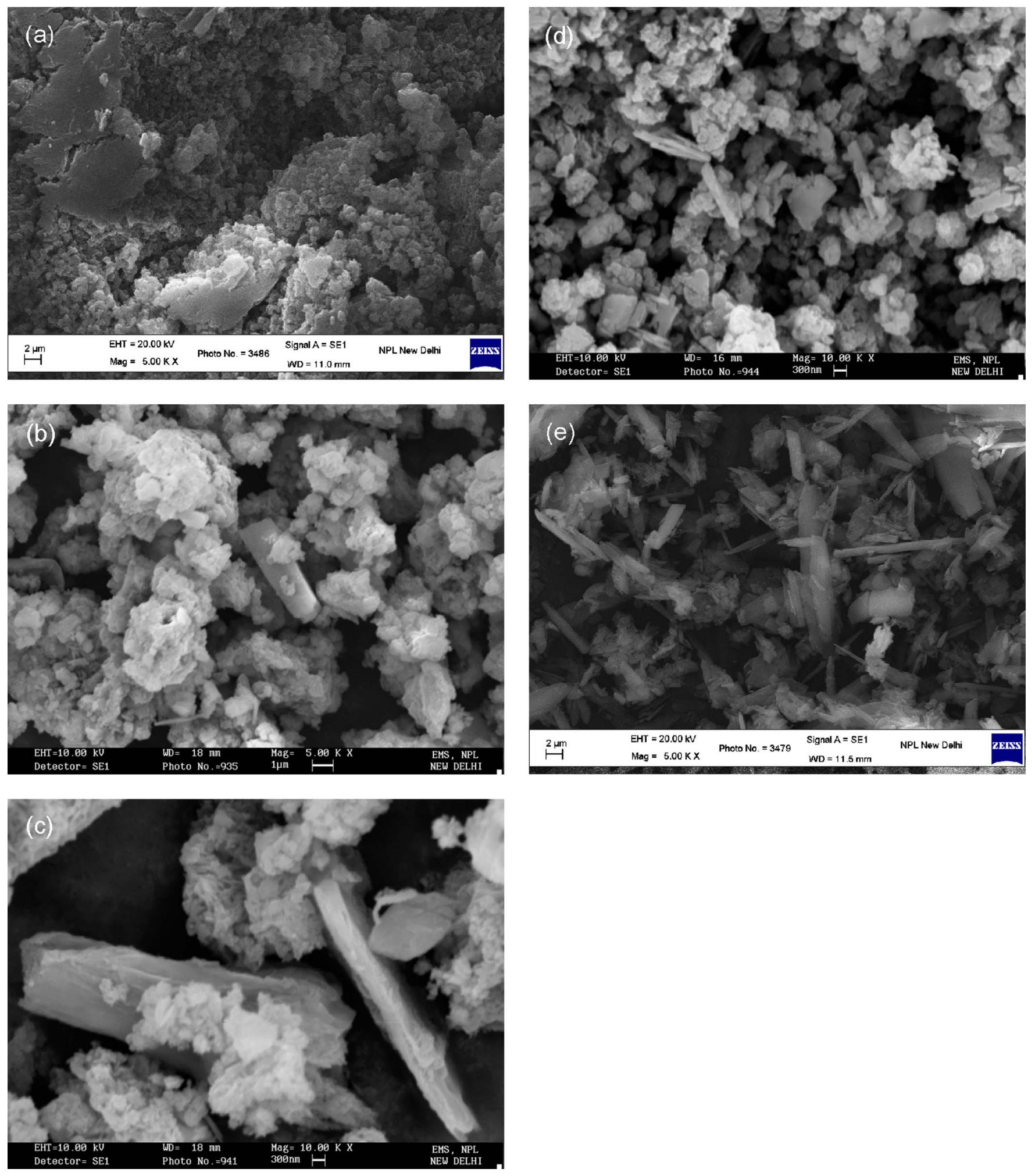

FIG. 1. (Color online) Scanning electron micrographs depicting varying crystallite domains of PDMA samples synthesized by controlled oxidant polymerization: (a) PDMA1, (b) PDMA2, (c) PDMA3, (d)PDMA4, and (e) PDMA5

$$
E_{A}=\left[\frac{-\partial \ln \sigma}{\partial\left(1 / k_{B} T\right)}\right],
$$

where $k_{B}$ is the Boltzmann's constant. We write the expression for de conductivity as

$$
\sigma_{\mathrm{dc}}=\sigma_{0}^{\prime} \exp \left[\frac{-\left(E_{C}-E_{F}\right)}{k_{B} T}\right],
$$

where $\sigma_{0}^{\prime}$ is a pre-exponential factor and $\left(E_{C}-E_{F}\right)$ is the band gap between the conduction band and the Fermi level. If it is temperature dependent, then the activation energy can be written as 


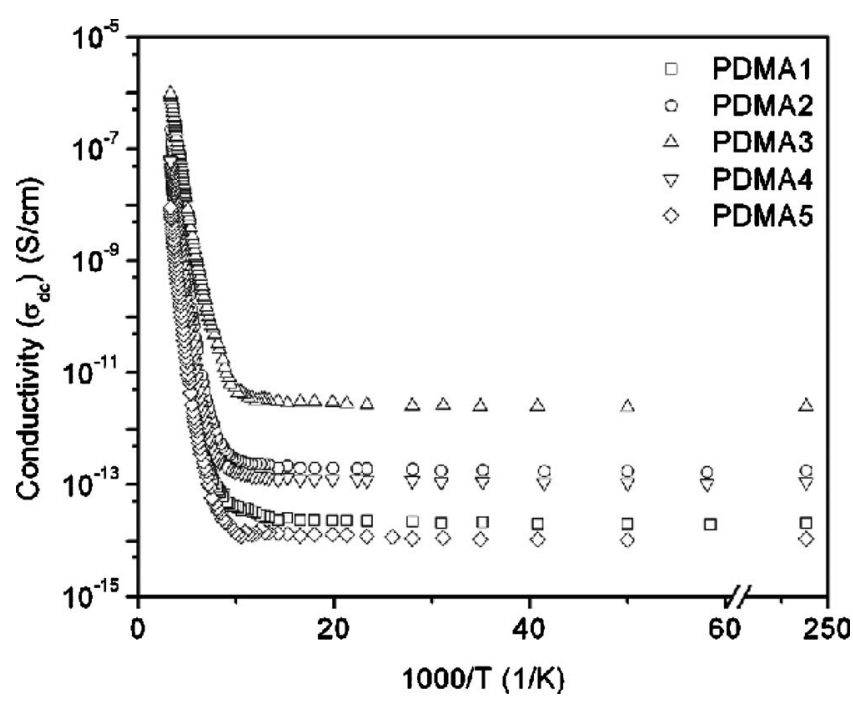

FIG. 2. Variation in de conductivity $\left(\sigma_{\mathrm{dc}}\right)$ as a function of reciprocal temperature in the range 6-303 $\mathrm{K}$ for different PDMA samples.

$$
E_{A}=\left(E_{C}-E_{F}\right)-T\left[\frac{\partial}{\partial T}\left(E_{C}-E_{F}\right)\right] .
$$

For a temperature dependent energy gap, the activation energy will be

$$
E_{A}=E_{g}-T\left(\partial E_{g} / \partial T\right)-\left(E_{F}-E_{V}\right),
$$

here the term $\left(E_{F}-E_{V}\right)$ in Eq. (4) is assumed to be temperature independent. In order to account for the temperature dependence of activation energy [Fig. 3(a)], the temperature dependence of energy gap has to be superlinear. If we assume that $E_{g}$ in Eq. (4) has the general form $A T^{n}$, where $A$ and $n$ are disposable constants, then from Eq. (4)

$$
E_{A}=A T^{n}(1-n)-\left(E_{F}-E_{V}\right) .
$$

The slope of a plot of $E_{A}$ versus $T$ is then given by

$$
\frac{\partial}{\partial T}\left(E_{A}\right)=A(1-n) T^{n-1} .
$$

To explain the experimental results [Fig. 3(a)], it would require that $(1-n)$ and $(n-1)$ be both positive simultaneously which is clearly not possible. Therefore, any such temperature dependence of the band gap or energy gap has been ruled out. The failure of the simple band conduction model to explain the observed temperature dependence of dc conductivity further supports that the hopping transport $\left(\sigma_{H}\right)$ may be the dominant mechanism of charge transport in the present investigation. In the hopping transport process, each state can have only one electron in each spin direction. If the localization is very strong, an electron will jump to the nearest state and the nearest neighbor hopping conductivity will be proportional to Boltzmann's factor $\exp \left(-W / k_{B} T\right)$, where $W$ is the energy difference between two states. If the localization is less strong the electron will jump to the sites for which the activation energy is smaller. But which can reside further away and then the conduction occurs by VRH conduction. The localization of charge carriers gives rise to the formation of polarons. As chains of conjugated polymers are randomly distributed, it behaves similar to a three-
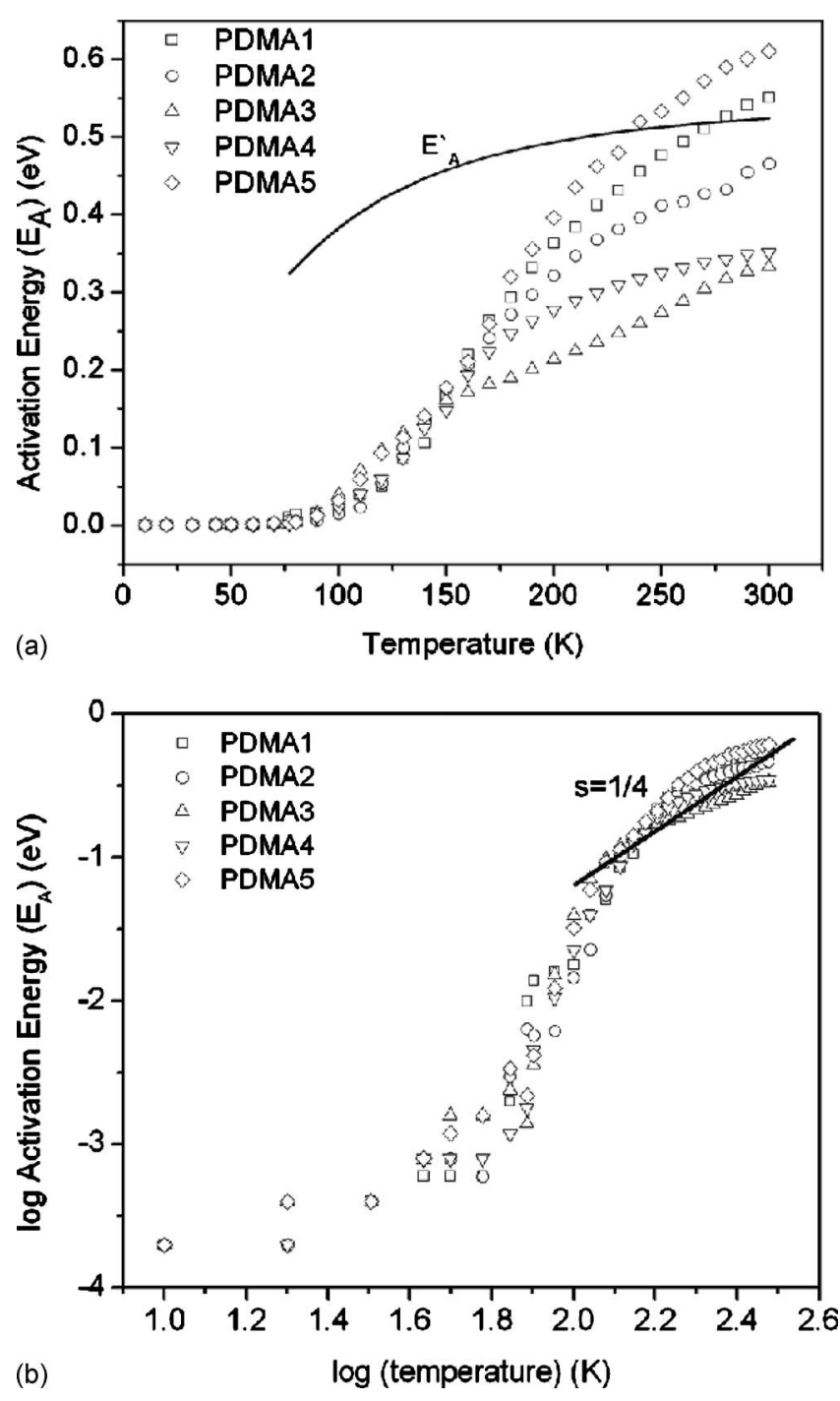

FIG. 3. (a) Plot of activation energy $\left(E_{A}\right)$ as a function of temperature $(6-300 \mathrm{~K})$ and (b) plot of $\log E_{A}$ vs $\log T$ for different PDMA samples. The values of $E_{A}^{\prime}$ calculated by using Eq. (9) for PDMA1 are shown by a solid line as a representative result in Fig. 3(a). The slope $s=1 / 4$ is shown in Fig. 3 (b) in the temperature range $100-300 \mathrm{~K}$.

dimensional (3D) system. In this situation, it can be stated that each time the charge carrier moves between the polymer chains, the charge carrier just below the Fermi level jumps to a state just above it with the energy $W$. Then the charge carrier gets transferred from one chain to another (adjacent chain) and the wave function overlaps that of the first chain. $12,13,16$

Henceforth, the hopping conductivity, as predicted by Mott and Davis, ${ }^{12}$ is of the form

$$
\sigma_{H}=\sigma_{0} \exp \left\{-\left(\frac{T_{0}}{T}\right)^{s}\right\}
$$

where $\sigma_{0}$ and $T_{0}$ are constants. $T$ is the temperature and $s$ $=1 / d+1$, where $s$ is the dimensionality. A plot of $\log \sigma_{H}$ versus $(1 / T)^{s}$ should give a straight line for proper value of $s$. It is seen from Fig. 2 that the conductivity data tends to saturate below $\sim 100 \mathrm{~K}$ for all the samples. Fig. 2 shows the applicability of 3D-VRH model above $100 \mathrm{~K}$ which indicates that the charge transport occurs by phonon assisted 

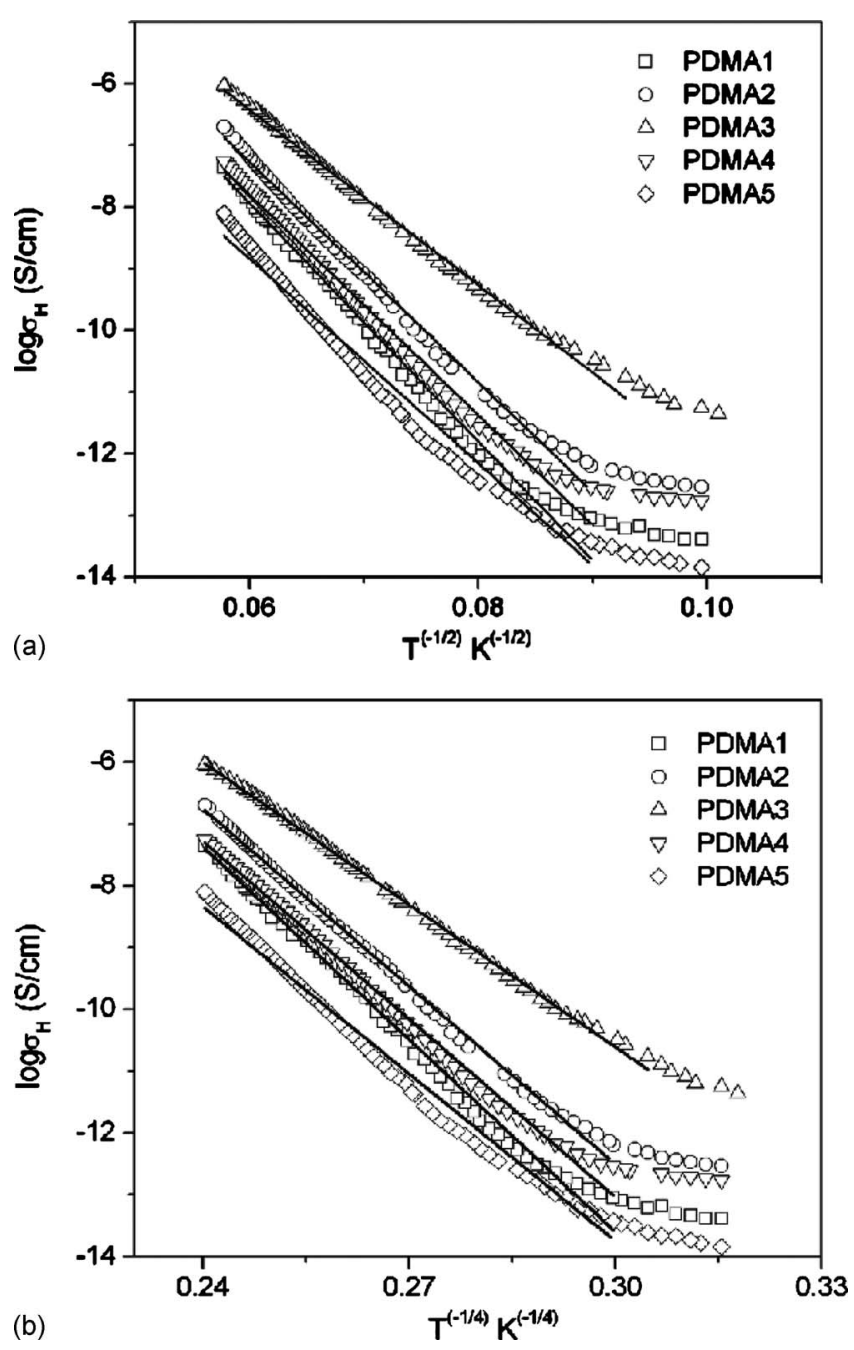

FIG. 4. The hopping conductivity $\left(\sigma_{H}\right)$ plotted as functions of (a) $T^{-1 / 2}$ and (b) $T^{-1 / 4}$ in the temperature range of $100-303 \mathrm{~K}$.

hopping which are thermally activated jumps between the localized sites. At low temperatures below $100 \mathrm{~K}$ (Fig. 2) the conductivity becomes less activated type and the mechanism of charge transport can be attributed to the tunneling mechanism reported ${ }^{16,17}$ earlier for other conjugated polymers. In the present investigation (Fig. 2), two distinct mechanisms of charge transport are dominating, one in the lower (below 100 $\mathrm{K})$ and other in the higher (100-303 K) temperature regions.

Therefore, the dc conductivity data in the temperature range 100-303 K has been examined in terms of Mott's VRH model. ${ }^{12}$ For this the dc conductivity data of all the samples has been plotted as functions of $T^{-1 / 2}$ and $T^{-1 / 4}$ in the temperature range 100-303 K in Figs. 4(a) and 4(b). The linearity factor of $T^{-1 / 2}$ and $T^{-1 / 4}$ plots are given in Table II. It is seen from Table II that the linearity factor for $T^{-1 / 4}$ gives the best fit. The plots [Fig. 4(b)] in the temperature region (100$303 \mathrm{~K}$ ), where Eq. (10) is valid should give activation energy as Eq. (1) and can be correlated to the parameters of Eq. (7) by the following expression:

$$
E_{A}=s k_{B} T_{0}\left(\frac{T_{0}}{T}\right)^{s-1} \text {. }
$$

It is evident from Eq. (8) that a plot of $\log E_{A}$ versus $\log T$ should yield a straight line of slope- $(s-1)$. The plot of $\log$ of activation energy as a function of $\log T$ is given in Fig. 3(b). It is evident from Fig. 3(b) that the activation energy shows systematic decrease with decreasing temperature. A straight line corresponding to $s=1 / 4$ is also shown in Fig. 3(b). It appears that the value of $s=1 / 4$ may satisfy the variation in activation energy with temperature in the range 100-303 K. This further indicates that the VRH mechanism of type $T^{-1 / 4}$ may dominate the mechanism of conduction in PDMA in the temperature range of 100-303 K. The temperature dependent activation energy can also be qualitatively explained if polaronic hopping is considered. ${ }^{25}$ For an ordered materials having polaronic conduction, multiphonon processes are involved. These processes are gradually replaced at lower temperatures by the processes in which the only contribution to the jump frequency of polarons is due to single optical phonon absorption or emission. The variation in activation energy for such a process is given ${ }^{13,25}$ by the equation

$$
\frac{E_{A}^{\prime}}{E_{A}}=\frac{\left[\tanh \left(\frac{\hbar \omega_{0}}{4 k_{B} T}\right)\right]}{\left(\frac{\hbar \omega_{0}}{4 k_{B} T}\right)},
$$

where $E_{A}^{\prime}$ is the calculated activation energy at different temperatures, $\omega_{0}=2 \pi v_{0}, \hbar=\mathrm{h} / 2 \pi$, and $E_{A}$ is the room temperature activation energy. As a representative result, the theoretical plot for $E_{A}^{\prime}$ from Eq. (9) is shown as a solid line in Fig. 3(a) for sample PDMA1. The polaronic hopping conduction can give temperature independent activation energy where the multiphonon process dominates. However, the temperature dependence of $E_{A}^{\prime}$ in Fig. 3(a) rules out the above possibility, confirming the polaronic conduction through VRH, where single phonon processes are involved, in samples of

TABLE II. Various Mott's parameters for the samples of PDMA along with linearity factors of $T^{-1 / 2}$ and $T^{-1 / 4}$

\begin{tabular}{|c|c|c|c|c|c|c|}
\hline \multirow[b]{2}{*}{ Sample number } & \multicolumn{2}{|c|}{ Linearity factor } & \multirow{2}{*}{$\begin{array}{c}T_{0} \\
(\mathrm{~K})\end{array}$} & \multirow{2}{*}{$\begin{array}{c}N\left(E_{F}\right) \\
\left(\mathrm{cm}^{-3} \mathrm{eV}^{-1}\right)\end{array}$} & \multirow{2}{*}{$\begin{array}{l}R \\
(\AA)\end{array}$} & \multirow{2}{*}{$\begin{array}{c}W \\
(\mathrm{eV})\end{array}$} \\
\hline & $T^{-1 / 2}$ & $T^{-1 / 4}$ & & & & \\
\hline PDMA 1 & 0.9901 & 0.9938 & $3.262 \times 10^{9}$ & $2.988 \times 10^{17}$ & 129.2 & 0.369 \\
\hline PDMA 2 & 0.9947 & 0.9977 & $2.372 \times 10^{9}$ & $4.108 \times 10^{17}$ & 119.3 & 0.342 \\
\hline PDMA 3 & 0.9959 & 0.9985 & $9.804 \times 10^{8}$ & $9.942 \times 10^{17}$ & 95.7 & 0.274 \\
\hline PDMA 4 & 0.9911 & 0.9951 & $2.387 \times 10^{9}$ & $4.082 \times 10^{17}$ & 119.5 & 0.342 \\
\hline PDMA 5 & 0.9742 & 0.9833 & $1.862 \times 10^{9}$ & $5.233 \times 10^{17}$ & 112.3 & 0.321 \\
\hline
\end{tabular}
plots [Figs. 4(a) and 4(b)]. 
PDMA. Based on audio-microwave frequency conductivity data on PANI it has been proposed that the charge carrier hopping among fixed polaron and bipolaron sites is the primary mechanism of charge transport in lightly protonated emeraldine base. However, at higher doping levels the polarons are arranged to form a polaron lattice with metal like transport within partially filled polaron energy band. ${ }^{6,7}$ The mechanism of charge transport in PDMA is also similar to that of unsubstituted PANI. Due to introduction of methoxy groups in aromatic ring, a steric repulsion between $\mathrm{H}$ and bulky- $\mathrm{OCH}_{3}$ group is produced which brings about torsion in benzenoid moiety leading to reduction in extent of orbital overlap and increase in the band gap. Hence the activation energy (Table I) of PDMA is more than that observed in case of unsubstituted PANI. ${ }^{6,7,9,10}$

Eqs. (7) and (8) give the slope $T_{0},{ }^{12,13,19}$ of the plots of $\log \sigma_{\mathrm{dc}}$ versus $T^{-1 / 4}$ [Fig. 4(b)] as

$$
T_{0}=\frac{\lambda \alpha^{3}}{k_{B} N\left(E_{F}\right)},
$$

where $k_{B}$ is the Boltzmann's constant, $T_{0}$ is the characteristic temperature, $\lambda$ is the dimensionless constant and is assumed to be $\sim 18.1,{ }^{13,19,26} N\left(E_{F}\right)$ is the density of states at the Fermi level, and $\alpha\left(=1 / r_{p}\right)$ is the coefficient of exponential decay of the localized states involved in the hopping transport process. The accurate estimation of $T_{0}$ from Fig. 4(b) provides the estimation of $N\left(E_{F}\right)$, i.e., the density of states at the Fermi level, which in turn provides the estimation of average hopping distance $R$ and average hopping energy $W$. The other constant $\sigma_{0}$ in Eq. (7) is given by

$$
\begin{aligned}
& \sigma_{0}=e^{2} R^{2} \nu_{p h} N\left(E_{F}\right), \\
& \text { where } R=\left[\frac{9}{8 \pi \alpha k_{B} T N\left(E_{F}\right)}\right]^{1 / 4},
\end{aligned}
$$

is the average hopping distance between the two sites. $\nu_{p h}$ is a phonon frequency $\left(\sim 10^{13} \mathrm{~Hz}\right)$ and can be obtained from Debye temperature $\theta_{\mathrm{D}}{ }^{27,28}$ The average hopping energy $W$ can be estimated by knowing the average hopping distance $R$ and the density of states at the Fermi level $N\left(E_{F}\right)$ by the following relation:

$$
W=\frac{3}{4 \pi R^{3} N\left(E_{F}\right)} .
$$

The values of various Mott's parameters $T_{0}, N\left(E_{F}\right), R$, $W$, for all the samples of PDMA have been evaluated by using Eqs. (10)-(13) and are reported in Table II. It has been assumed $^{13,19,26,29}$ that the electron wave function localization length, i.e., $\alpha^{-1}\left(r_{p}\right)$ is equal to the width of poly(omethoxyaniline) monomer unit, i.e., $\sim 5.995 \AA{ }^{24}$ since the electrons are always at least delocalized to the extent of the $\pi$-orbitals on the monomer units. This parameter yields the density of states at the Fermi level; $\left[N\left(E_{F}\right)\right]$ on the assumption that the wave function localization length remains independent of temperature and conductivity. The values of $T_{0}$ has been estimated from the slope of $\log \sigma_{\mathrm{dc}}$ versus $T^{-1 / 4}$ [Fig. 4(b)]. Similarly $R$ and $W$ for all samples have been evaluated by using Eqs. (12) and (13). The values of $T_{0}, R$,
$W$, and $N\left(E_{F}\right)$ are given in Table II. It can be seen from Table II that the density of states at the Fermi level; $N\left(E_{F}\right)$ of all the PDMA samples lies in the range $\sim 10^{17}-10^{18} \mathrm{~cm}^{-3} \mathrm{eV}^{-1}$ which is in good agreement with the values reported earlier for other conjugated polymers. ${ }^{6,10,13-19}$ Our results are consistent with the Mott's requirement that $\alpha R \gg 1$ and $W$ $\gg k_{B} T$ for conductivity by hopping to distant sites (Table II). The temperature dependent activation energy [Fig. 3(a)] and evaluated values of Mott's parameters (Table II) suggest that the Mott's 3D-VRH model successfully explains the mechanism of charge transport in PDMA in the temperature range 100-303 K.

\section{CONCLUSIONS}

The measured dc conductivity of PDMA samples in the temperature range $6-303 \mathrm{~K}$ is the sum of hopping and tunneling conductivities. The tunneling contribution is dominant only below $100 \mathrm{~K}$. The temperature dependence of dc conductivity in the range 100-303 $\mathrm{K}$, where hopping conduction mechanism dominates, is well represented by Mott's 3DVRH. The evaluated values of Mott's parameters of PDMA samples are in good agreement with those reported earlier for other conjugated polymers.

\section{ACKNOWLEDGMENTS}

The authors are grateful to Director, National Physical Laboratory, New Delhi for his permission to publish this work. The authors are thankful to Dr. H. K. Singh and Mr. K. N. Sood of National Physical Laboratory, New Delhi for cooperation. Two of us (A.K. and R.S.) are thankful to Council of Scientific and Industrial Research (CSIR), Government of India, New Delhi for the award of Junior Research Fellowship and Emeritus Scientist Fellowship, respectively.

${ }^{1}$ Handbook of Conducting Polymers, edited by T. A. Skotheim, R. L. Elsenbaumer, and J. R. Reynolds (Madison Avenue, New York, USA, 1998), Chap. 5.

${ }^{2}$ D. C. Trivedi, in Handbook of Organic Conductive Molecules and Polymers, edited by H. S. Nalwa (Wiley, New York, 1997), Vol. 2, Chap. 12, pp. 505-572, and references therein.

${ }^{3}$ P. Yin and P. A. Kilmartin, Curr. Appl. Phys. 4, 141 (2004).

${ }^{4}$ S. E. Mavundla, G. F. Malgas, P. Baker, and E. I. Iwuohab, Electroanalysis 20, 2347 (2008).

${ }^{5}$ B. Palys, A. Kudelski, A. Stankiewicz, and K. Jackowska, Synth. Met. 108, 111 (2000).

${ }^{6}$ F. Zuo, M. Angelopoulos, A. G. MacDiarmid, and A. J. Epstein, Phys. Rev. B 39, 3570 (1989).

${ }^{7}$ H. H. S. Javadi, K. R. Cromack, A. G. MacDiarmid, and A. J. Epstein, Phys. Rev. B 39, 3579 (1989).

${ }^{8}$ J. M. Ginder, A. F. Ritcher, A. G. MacDiarmid, and A. J. Epstein, Solid State Commun. 63, 97 (1987).

${ }^{9}$ J. F. Rouleau, J. Goyette, T. K. Bose, R. Singh, and R. P. Tandon, Phys. Rev. B 52, 4801 (1995).

${ }^{10}$ R. Singh, V. Arora, R. P. Tandon, and S. Chandra, Polymer 38, 4897 (1997), and references therein.

${ }^{11}$ W. Xue, H. Qiu, K. Fang, J. Li, J. Zhao, and M. Li, Synth. Met. 156, 833 (2006).

${ }^{12}$ N. F. Mott and E. A. Davis, Electronic Processes in Non-Crystalline Materials, 2nd ed. (Oxford University Press, London, 1979).

${ }^{13}$ R. Singh, A. K. Narula, R. P. Tandon, A. Mansingh, and S. Chandra, J. Appl. Phys. 79, 1476 (1996).

${ }^{14}$ R. Singh, A. K. Narula, R. P. Tandon, A. Mansingh, and S. Chandra, J. Appl. Phys. 81, 3726 (1997).

${ }^{15}$ R. Singh and A. K. Narula, J. Appl. Phys. 82, 4362 (1997). 
${ }^{16}$ R. K. Singh, J. Kumar, R. Singh, R. Kant, R. C. Rastogi, S. Chand, and V. Kumar, New J. Phys. 8, 112 (2006).

${ }^{17}$ R. Singh, A. Kaur, K. L. Yadav, and D. Bhattacharya, Curr. Appl. Phys. 3, 235 (2003).

${ }^{18}$ R. K. Singh, J. Kumar, R. Singh, R. Kant, S. Chand, and V. Kumar, Mater. Chem. Phys. 104, 390 (2007).

${ }^{19}$ R. Singh, J. Kumar, R. K. Singh, S. Chand, and V. Kumar, J. Appl. Phys. 100, 016106 (2006).

${ }^{20}$ S. Capaccioli, M. Lucchesi, D. Prevosto, and P. A. Rolla, Phys. Status Solidi C 1, 148 (2004).

${ }^{21}$ M. C. Gupta and S. S. Umare, Macromolecules 25, 138 (1992).

${ }^{22}$ R. Singh, R. K. Singh, J. Kumar, R. Kant, and V. Kumar, J. Polym. Sci., Part B: Polym. Phys. 48, 1047 (2010).
${ }^{23}$ R. K. Singh, A. Kumar, K. Agarwal, D. Dwivedi, K. N. Sood, and R. Singh (to be published).

${ }^{24}$ P. F. Van Hutten and G. Hadzioannou, in Handbook of Organic Conductive Molecules and Polymers, edited by H. S. Nalwa (Wiley, New York, 1997), Vol. 3, Chap. 1, pp. 1-86.

${ }^{25}$ T. Holstein, Ann. Phys. 8, 343 (1959).

${ }^{26}$ D. K. Paul and S. S. Mitra, Phys. Rev. Lett. 31, 1000 (1973).

${ }^{27}$ R. Singh, J. Kumar, R. K. Singh, R. C. Rastogi, and V. Kumar, New J. Phys. 9, 40 (2007).

${ }^{28}$ R. Singh, R. P. Tandon, and S. Chandra, J. Phys.: Condens. Matter 5, 1313 (1993).

${ }^{29}$ D. S. Maddison and T. L. Tansley, J. Appl. Phys. 72, 4677 (1992). 